Editorial

\title{
Dysphagia: A Symptom to Be Controlled
}

\author{
Geraldo P. Jotz ${ }^{1}$ \\ 1 Professor and Chairman of the Morphological Sciences Department, \\ Universidade Federal do Rio Grande do Sul, Porto Alegre, RS, Brazil \\ Int Arch Otorhinolaryngol 2014;18:90-91.
}

We would like to highlight the article titled "Analysis of the Level of Dysphagia, Anxiety, and Nutritional Status before and after Speech Therapy in Patients with Stroke." This study analyzed the nutritional status, oral ingestion, dysphagia level, and anxiety before and after speech therapy in patients diagnosed with stroke. Twelve patients (mean age 64.6 years) without cognitive disorders and a prior diagnosis of hemorrhagic or ischemic stroke were evaluated. All tests were conducted before and after speech therapy. A statistically significant improvement in the level of dysphagia $(p<0.017)$ and oral intake $(p<0.003)$ was observed after speech therapy. Although considerable improvement was observed in the patients' level of anxiety and nutritional status, the improvement was not statistically significant.

Dysphagia is not defined as a disease but as a series of symptoms caused by an underlying illness. Dysphagia is a common disorder normally observed in elderly patients.

Twenty years ago, the treatment of patients with swallowing disorders was completely different from the current approach. During that period, the only concern was to maintain a supply route, regardless of the implications on the quality of the patient's life. Salivation issues were not highlighted as they are today. Medical professionals and phonoaudiologists did not have the expertise and foresight to determine the impact that these changes would have on the near future.

Literature reveals that 45 to $65 \%$ of patients with cerebral vascular accidents (CVA; i.e., strokes) face difficulties during the first 6 months after this event. ${ }^{1-3}$ Although many of these patients partially or completely recover their swallowing ability, approximately $50 \%$ continue to inhale, and $25 \%$ of the latter die due to aspiration pneumonia in the first year after CVA.

A large number of individuals are afflicted with Parkinson disease due to the increased number in elderly people, and this disease is commonly associated with speech and swallowing disorders. ${ }^{4}$ Approximately 50\% of the patients reported symptoms of dysphagia 5 years before the onset of Parkinson disease. ${ }^{5}$ Dysphagia is also common in people with Alzheimer disease, although the mechanism is yet to be ascertained. ${ }^{6}$
Recently, a study of three patients suffering from vascular decompression of the medulla oblongata described significant relief from symptoms of dysphagia. ${ }^{7}$

The most common cause of swallowing disorders in patients with neck and head cancer is either the disease itselfwith involvement of the oral cavity, pharynx, and/or larynx-or the treatment, which consists of extensive removal of mucosal tissue from the oral cavity, pharynx, and/or esophagus with or without reconstruction of neighboring or distant tissues.

Swallowing disorders have a significant impact on public health mainly due to the nutritional aspects that may lead to obesity. ${ }^{8}$ Several medications are also known to cause swallowing disorders.

Dysphagia continues to be an important symptom with an ill-defined epidemiology. Some studies suggest a 16 to $22 \%$ prevalence rate. Other studies state that dysphagia will become a significant public health problem with increase in age. Few studies are available regarding the impact of dysphagia on the patients' quality of life.

It is well known that muscle composition changes as age increases, and the components of and coordination between the motor units decrease, resulting in shape alteration, decreased number of fast-twitch fibers, and reduced number of enzymes required for muscular contraction. This results in a change in the strength and mobility of phonoarticular organs triggered by decreased amount of saliva, delay in chewing and swallowing, changes in food consistency, presence of oral stasis in pyriform sinuses, and delay and/or absence of coordination in bolus transition.

Pulmonary aspiration is one of the most severe complications faced by patients with dysphagia. Therefore, an oral diet should be administered with food consistency that prevents aspiration and dehydration. ${ }^{9}$ For these patients, food intervention depends on the diet prescribed to each patient with the aim of minimizing the risks and discomfort while promoting health and quality of life.

Currently as well as in the future, swallowing disorders will occur more frequently as the population ages. Both

Copyright $@ 2014$ by Thieme Publicações License terms Ltda, Rio de Janeiro, Brazil

\author{
Address for correspondence \\ Geraldo P. Jotz, Morphological \\ Sciences Department, Universi-
dade Federal do Rio Grande do \\ Sul, Porto Alegre, RS, Brazil \\ DOI http://dx.doi.org/ \\ 10.1055/s-0034-1372694. \\ ISSN 1809-9777.
}


swallowing and talking are and will be essential to human life in the next millennium. Patients with dysphagia may suffer from serious or severe complications, regardless of socioeconomic and social factors. Diagnosis of these symptoms is essential as the condition of the patient could deteriorate rapidly leading to death.

In 2014, the International Archives of Otorhinolaryngology awarded two systematic reviews that were published in our journal in 2013 with the aim to highlight articles that state the basis and concepts of universal knowledge.

The studies selected by the editors were:

1: Carmo MP, Costa NTO, Momensohn-Santos TM. Tympanometry in Infants: A Study of the Sensitivity and Specificity of $226-\mathrm{Hz}$ and $1,000-\mathrm{Hz}$ Probe Tones. Int Arch Otorhinolaryngol 2013; 17(4): 395-402.

2: Balata PMM, Silva HJ, Moraes KJR, Pernambuco LA, Moraes SRA. Use of surface electromyography in phonation studies: an integrative review. Int Arch Otorhinolaryngol 2013; 17(3): 329-339.

We congratulate all the authors!

\section{Geraldo Pereira Jotz}

Editor-in-Chief

International Archives of

Otorhinolaryngology

\section{References}

1 Gordon C, Hewer R, Wade D. Dysphagia in acute stroke. Brit Med J 1987;295:411-414

2 Kidd D, Lawson J, Nesbitt R, MacMahon J. Aspiration in acute stroke: a clinical study with videofluoroscopy. Quarterly J Med 1993;86:825-829

3 Mann G, Hankey G, Cameron D. Swallowing function after stroke: progress and prognostic factors at 6 months. Stroke 1999; 30:744-748

4 Dray T, Hillel A, Miller R. Dysphagia caused by neurologic deficits. Otolaryngol Clin North Am 1998;31:507-524

5 Park C, O'Neill PA. Management of neurological dysphagia. Clin Rehabilit 1994;8:166-174

6 Ratnaike R. An Essential Guide to Geriatric Practice. New York, NY: McGraw Hill; 2002

7 Nakahara Y, Kawashima M, Matsushima T, et al. Microvascular decompression surgery for vertebral artery compression of the medulla oblongata: three cases with respiratory failure and/or dysphagia. W Neurosurg 2014; in press.

8 Gustafsson B, Tibbling L. Dysphagia: an unrecognized handicap. Dysphagia1991;6:193-199

9 Karagiannis M, Karagiannis TC. Oropharyngeal dysphagia, free water protocol and quality of life: an update from a prospective clinical trial. Hellenic J Nuclear Med 2014;(Suppl):26-29 